\title{
Assessing sensitivity to Condition $A$ in the case of Chinese reflexives
}

\author{
Jun Lyu \& Elsi Kaiser*
}

\begin{abstract}
There are two reflexives in Mandarin Chinese, ziji ('self') and ta-ziji ('s/he-self'). It is often assumed that ziji can be bound by a non-local antecedent while ta-ziji cannot. This is because ziji can be used as an exempt anaphor licensed by discourse-pragmatic conditions. However, prior research shows that, in contexts without perspectival cues, ziji tends to be interpreted as a 'regular' syntactically bound reflexive, exhibiting a similar locality bias as $t a-z i j i$. However, prior studies comparing the locality biases of ziji and ta-ziji present divergent results. In this study, we report two forced choice judgment experiments to assess which reflexive, $z i j i$ or $t a-z i j i$, exhibits a stronger locality bias. Overall, our results fit better with claims that in local contexts, $t a-z i j i$ is preferred over $z i j i$; we find no clear evidence of ziji being preferred over ta-ziji in local contexts. Our results are compatible with the idea that $t a-z i j i$, rather than $z i j i$, is more constrained by Condition A.
\end{abstract}

Keywords. Mandarin Chinese, reflexive, recency, locality, classifiers

1. Introduction. There are two types of reflexives in Mandarin Chinese (henceforth "Chinese"), a bare reflexive ziji ('self') and a compound reflexive ta-ziji ('s/he-self') which is similar to English himself/herself in morphological composition. According to Condition A of the Binding Theory (Chomsky 1981), a reflexive must be bound within its local domain. However, it was soon realized that this description of the behavior of reflexives, even in English, falls short of accounting for some exceptional cases. In fact, different views on reflexive binding predate the Binding Theory (e.g., Postal 1971, Kuno 1972). Most relevant for this paper, Kuno (1987) proposes that the notion of point of view - also referred to as perspective-taking - is crucial to the interpretation of anaphora in English (also see e.g., Zribi-Hertz 1989). Indeed, a large body of literature shows that perspective-taking plays a pivotal role in licensing long-distance (LD) binding of so-called "exempt anaphors" (e.g., Pollard \& Sag 1992, Reinhart \& Reuland 1993 and many others), not only in English but also in other languages (e.g., Sells 1987).

In particular, it has been argued that Chinese ziji exhibits sensitivity to perspective centers (e.g., Huang \& Liu 2001, Huang et al. 2009, Wang \& Pan 2015, Charnavel et al. 2017) - people whose points of view or perspectives the speaker takes. ${ }^{1}$ Under this view, LD binding of ziji is licensed if the non-local antecedent is a perspective center. Thus, in (1), ziji can be bound by the matrix subject Zhangsan if a speaker takes Zhangsan's perspective.

$$
\begin{aligned}
& \text { Zhangsan }_{\mathrm{i}} \text { zhidao Lisi }_{\mathrm{j}} \text { xihuan ziji } \mathrm{i}_{\mathrm{ij}} \text {. } \\
& \text { Zhangsan know Lisi like self }
\end{aligned}
$$

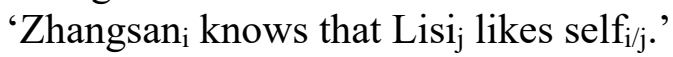

\footnotetext{
* The authors would like to thank the audience at the $95^{\text {th }}$ Annual Meeting of the Linguistic Society of America for helpful comments and suggestions. Authors: Jun Lyu (junlyu@usc.edu) \& Elsi Kaiser (emkaiser@usc.edu), University of Southern California.

1 An often-cited phenomenon to support this view is the "blocking effect" where a local $11^{\text {st }} / 2^{\text {nd }}$-person pronoun blocks LD binding (e.g., Huang et al. 1985, Pan 1997, 2001). This is because a $1^{\text {st }} / 2^{\text {nd }}$-person pronoun encodes the perspective of the speaker/comprehender whose perspective is more privileged than that of a $3^{\text {rd }}$-person NP.
} 
In contrast, it is claimed that ta-ziji is not sensitive to perspective centers and, unlike ziji, does not allow LD binding when the local antecedent is a human, shown in (2) taken from Pan (1998: 781):

(2) Zhangsan Zhidao Lisi $_{j}$ xihuan ta-ziji $*_{i} / j$.

Zhangsan know Lisi like s/he-self

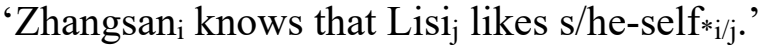

However, non-local binding of ta-ziji is still acceptable if the local antecedent is a non-human as in (3) (Pan 1998: 782). This is attributed to ta-ziji being sensitive to the antecedent's prominence in terms of the animacy hierarchy (Chou 1992: human $>$ non-human $>$ inanimate). Thus, if the non-local antecedent is more prominent (higher ranked on animacy) than the local one, LD binding is allowed. This leads Pan (1998) to conclude that ta-ziji is not only constrained by Condition A but also by an animacy prominence constraint.

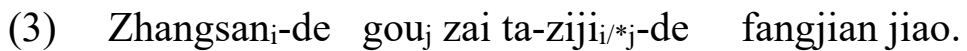

Zhangsan-GEN dog at s/he-self-GEN room bark

'Zhangsan ${ }_{\mathrm{i}}$ 's $\operatorname{dog}_{\mathrm{j}}$ is barking in $\mathrm{s} / \mathrm{he}-\mathrm{self}_{\mathrm{i} / \mathrm{j}}$ 's room.'

To summarize, the claims in prior work are that ziji allows LD binding when the non-local antecedent is a perspective center (regardless of the animacy of the local antecedent), while ta$z i j i$ allows LD binding only when the non-local antecedent outranks the local antecedent on the animacy hierarchy. Note that it is a common assumption in the literature on Chinese that local $z i j i$ and LD ziji show different linguistic properties (e.g., Pan 1997, Huang \& Liu 2001). Local $z i j i$ is governed by Condition A - it's a syntactic reflexive - while LD ziji is governed by discourse-pragmatic conditions (see e.g., Huang \& Liu 2001, Huang et al. 2009, Charnavel et al. 2017) - an exempt anaphor (However, see Charnavel (2020) for a recent analysis proposing a unification between syntactic and 'exempt' anaphora, based largely on data from French, but with crosslinguistic implications. In the present paper, we follow the assumption traditionally assumed in work on Chinese that distinguishes between two different linguistic entities for ziji. However, investigating an analysis along the lines of Charnavel (2020) is an important direction for future work). In contrast, ta-ziji can only be a syntactic reflexive as it does not show sensitivity to perspective centers.

2. Research questions. From the brief discussion above, one might be tempted to conclude that $t a-z i j i$ should show a stronger locality bias $^{2}$ than $z i j i$, as the latter can be optionally interpreted as an exempt anaphor. However, prior theoretical and experimental work does not support this conclusion. Reuland $(2001,2011)$ argues that it is more economical for anaphoric relations to be encoded in narrow syntax than on the level of discourse. In other words, given a choice between $z i j i$ as a syntactic reflexive (constrained by Condition A) or an exempt anaphor (licensed by the presence of a perspective center), the former is preferred.

Indeed, psycholinguistic studies on Chinese reflexives support this view because, in absence of perspectival/point-of-view cues, native speakers have a strong tendency to bind ziji to a local antecedent (e.g., Gao et al. 2005, Li \& Zhou 2010, Dillon et al. 2014, 2016, Jäger et al. 2015, Wang $2017 \mathrm{a}, \mathrm{b})$, suggesting that ziji tends to be used as a syntactic reflexive rather than an

\footnotetext{
${ }^{2}$ In this paper, we reserve the term "locality bias" to refer descriptively to the empirical phenomenon where a reflexive tends to refer to the local antecedent. Thus, we make a terminological distinction between the empirical phenomenon and theoretical constructs such as "locality constraint" or "Condition A," which presumably contributes to the locality bias.
} 
exempt anaphor. Thus, one non-trivial question is, since both ziji and ta-ziji tend to be interpreted as syntactic reflexives in stand-alone sentences, do these two reflexives exhibit the same degree of sensitivity to Condition A?

This question is a controversial one. In one study by Dillon et al. (2016) with sentences like $(4 a, b)$, it was found that while local binding of ziji in (4a) is more acceptable to native speakers than the non-local binding in (4b), there is no judgment difference between local and non-local binding for $t a-z i j i$. The results of Dillon and colleagues are in line with a hypothetical view that $z i j i$ as a syntactic reflexive is more constrained by Condition A than ta-ziji. This being said, we would like to explicitly point out that Dillon et al. do not interpret their results in this way, as the research focus of their paper is different. In the present paper, we explore further this hypothetical view (not explicitly espoused by Dillon et al. 2016).

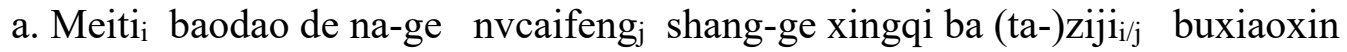
media report DE that-CL seamstress last-CL week BA (she-)self carelessly nongshang-le.

harm-PERF

'The seamstress $\mathrm{j}_{\mathrm{j}}$ that the media $\mathrm{i}_{\mathrm{i}}$ reported on carelessly harmed (she-)self $\mathrm{f}_{\mathrm{i} / \mathrm{j}}$ last week.'

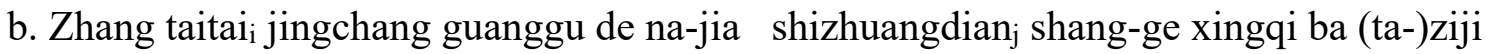
Mrs. Zhang often visit DE that-CL boutique last-CL week BA (she-)self buxiaoxin nongshang-le.

carelessly harm-PERF

'The boutique ${ }_{j}$ that Mrs. Zhang i $_{\mathrm{i}}$ often visited carelessly harmed (she-)self $\mathrm{i}_{\mathrm{i} / \mathrm{j}}$ last week.'

Thus, although both ziji and ta-ziji tend to be interpreted as syntactic reflexives, the extent to which they are constrained by Condition A seems to be different. This is consistent with a formspecific view on anaphora resolution (e.g., Kaiser \& Trueswell 2008) as Condition A has different impacts on the interpretation of ziji and ta-ziji. The findings of the study also fit well with a view proposed in a different subfield of linguistics, namely the "competitive mutual exclusion" account for synonyms (e.g., Lindsay \& Aronoff 2014, Aronoff 2019). The idea behind this account is that just like biological systems organize themselves through adaptation, synonyms also adapt to a crowded space through competition and mutual exclusion until they find their own functional and grammatical niches. Borrowing this idea, we can hypothesize that since ziji can be used both as a syntactic reflexive and an exempt anaphor, hence overlapping with the function of ta-ziji as a syntactic reflexive, ziji might be replacing ta-ziji as a syntactic reflexive, such that $t a-$ $z i j i$ is primarily reserved for its own niche (e.g., a contrastive reflexive). Thus, the findings of Dillon et al. (2016) could have important theoretical implications, which warrants a deeper look into the locality strength difference between ziji and ta-ziji.

But to our knowledge, there are few attempts to specifically assess the validity of the view that ziji shows stronger locality bias than ta-ziji. In addition to Dillon et al. (2016), only Lu (2011) and Wang (2017a, 2017b) directly and systematically compared the acceptability of local and non-local binding for ziji and ta-ziji. In two sentence acceptability judgment experiments, Wang (2017a,b) drew a similar conclusion as Dillon et al. (2016), although she pointed out that in her studies ta-ziji is situated in subject position and is likely a contrastive reflexive (Pan 1995, $\mathrm{Xu}$ 1999) which, she suggests, is exempt from Condition A (Pan 1998). In contrast, Lu (2011)'s investigation of the acceptability of ziji and ta-ziji in subject position found that ta-ziji shows a stronger locality bias than ziji. Note that in both Lu and Wang's studies, the critical reflexives are the subjects of an embedded clause and thus do not have a clausemate antecedent. This means 
that the so-called "local" antecedent is not local in a technical sense but simply linearly closer compared to the more distant matrix subject.

In the study reported in this paper, we approach the locality bias question from two new angles. First, instead of asking participants to judge sentence acceptability, we ask participants to choose which reflexive (ziji vs. ta-ziji) they prefer to use in local and non-local syntactic contexts. The working hypothesis is that if ziji has a stronger locality bias, then in local contexts, native speakers will prefer ziji over ta-ziji when asked to choose one or the other.

Second, in prior work there have been few efforts to tease apart syntactic locality from linear precedence (or recency). In prior studies, these two factors are often confounded. For example, in Ex.(1), Lisi is both a recent and a local antecedent (i.e., in the same finite clause as the reflexive). Contrast this with (5) where Lisi is only a local but not a recent antecedent. This is because its trace (or copy) inside the relative clause (RC) is a clausemate antecedent of (ta-)ziji. Lisi thus becomes the local antecedent by virtue of being the binder of its trace. In this paper, we call Lisi in (1) a non-strictly local antecedent (as it also linearly precedes the reflexive) and Lisi in (5) a strictly local antecedent (as it does not linearly precede the reflexive).

(5) Zhangsani yiwei [RC $\mathrm{t}_{\mathrm{j}}$ xihuan (ta-)ziji $\left.\mathrm{i}_{\mathrm{i} j \mathrm{j}} \mathrm{de}\right] \mathrm{Lisi}_{\mathrm{j}}$ bujian-le.

Zhangsan think like (he-)self DE Lisi disappear-PERF

'Zhangsan thinks that Lisi who likes (he-)self has disappeared.

The general aim of this study is to probe native speakers' preference for $z i j i$ or $t a-z i j i$ with strictly and non-strictly local antecedents. Our research questions are as follows:

1) Does ziji show a stronger locality bias than ta-ziji?

2) Do the locality biases of ziji and ta-ziji vary in syntactic structures with (non-)strictly local antecedents?

Investigating the above questions serves to not only shed light on which reflexive is more sensitive to Condition A, but also to test the validity of a form-specific view on anaphora resolution (e.g., Kaiser \& Trueswell 2008). Furthermore, the outcomes can inform us whether ziji and $t a-z i j i$ are organized into their own functional/grammatical niches as suggested above.

3. Experiment 1. In this experiment, we examine native speakers' preference for ziji or ta-ziji in sentences with strictly and non-strictly local antecedents. In the former, the local antecedent appears after the reflexive (see (5) above) and thus does not linearly precede the reflexive as is typical of the experimental designs in most prior studies.

3.1. PARTICIPANTS. Fifty-two participants self-identified as Mandarin native speakers (mean age $=21 ; \mathrm{SD}=3.89$ ) participated over the internet via Qualtrics.

3.2. MATERIALS AND DESIGN. The factors Structure type (complement clause (CP) vs. relative clause (RC)) and Antecedent position (local vs. non-local) were crossed in a factorial design. In the CP structure, the local antecedent is non-strictly local; but in the RC structure, the local antecedent is strictly local. The second factor is Antecedent position. As ziji and ta-ziji are typically only compatible with animate antecedents, the antecedent position is determined by the animacy of the intended antecedent. In other words, each target sentence only contains one compatible antecedent (the animate entity); the intended referent of the reflexive is not ambiguous. Local antecedents are NPs that are animate in local positions while non-local antecedents are animate in non-local positions. In this paper, we refer to sentences with local animate antecedents as the 
local context conditions, and sentences with animate non-local antecedents as the non-local context conditions.

We also included demonstrative-classifiers in CPs to make local NPs definite, on a par with local antecedents in $(6 \mathrm{c}, \mathrm{d})$ which are definite as they are modified by a restrictive RC. (In)animate local antecedents are paired with compatible classifiers (e.g., ming $\rightarrow$ a human; jia $\rightarrow$ a place/institution). For an example item, see (6). In all items, participants were given two options for what goes into the blank, as shown in Figure 1. On target trials, the two options were ziji-de ('self-GEN') and ta-ziji-de ('s/he-self-GEN') (similar to English 'his interview's purpose' or 'the purpose of his interview').

\section{a. CP structure/Local antecedent}

Diantai biaoshi na-ming jizhe gongbu-le___ caifang mudi. radio station say that-CL journalist reveal-PERF interview purpose 'The radio station said that the journalist had revealed the purpose of interview.'

\section{b. CP structure/Non-local antecedent}

Jizhe biaoshi na-jia diantai gongbu-le___ caifang mudi. journalist say that- CL radio station reveal-PERF interview purpose 'The journalist said that the radio station had revealed the purpose of ___ interview.'

\section{c. RC structure/Local antecedent}

Diantai biaoshi gongbu-le ___ caifang mudi de jizhe yinqi-le

Radio station say reveal-PERF_ interview purpose DE journalist provoke-PERF dajia-de buman.

everyone-GEN anger

'The radio station said that the journalist who revealed the purpose of voked everyone's anger.'

\section{d. RC structure/Non-local antecedent}

Jizhe biaoshi gongbu-le ___ caifang mudi de diantai yinqi-le

Journalist say reveal-PERF interview purpose DE radio station provoke-PERF dajia-de buman.

everyone-GEN anger

'The journalist said that the radio station which revealed the purpose of interview provoked everyone's anger.'

Participants read sentences like (6) and were asked to choose a reflexive (ziji-de 'self-GEN' or $t a-z i j i-d e$ 's/he-self-GEN') in its genitive form in a forced-choice task. They were expected to choose a reflexive that makes the sentence sound most natural. The presentation order of the answer choices (i.e., ziji-de and ta-ziji-de) was counterbalanced. See Figure 1 for an example of the forced choice question. Twenty-four sets of target items were distributed into 4 lists using a Latin-Square design. Targets were pseudo-randomized with 24 filler sentences which were also presented with a word missing. Participants were required to fill in the blank with an appropriate verb or adverb. 


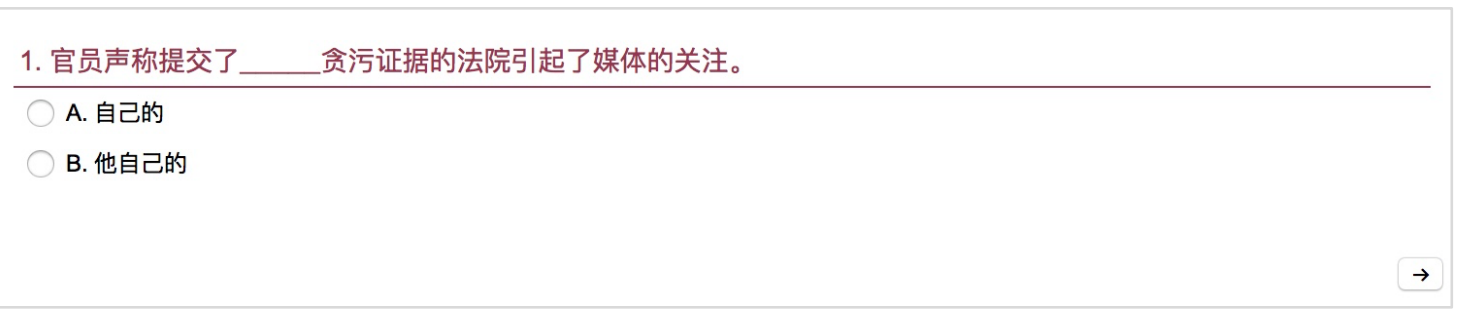

Figure 1. Example screenshot.

3.3. RESULTS. Table 1 shows participants' choices of ta-ziji versus ziji in local and non-local contexts in CP and RC structures (see Figure 2). We can see that in CP structures (where local antecedents are non-strictly local), participants showed an asymmetric choice pattern such that they preferred $t a-z i j i$ in local contexts but $z i j i$ in non-local contexts. But in RC structures (where local antecedents are strictly local), there is a general tendency to choose ziji.

\begin{tabular}{llll} 
Structure type & Antecedent position & ta-ziji & ziji \\
\hline $\mathrm{CP}$ & Local & 170 & 130 \\
& Non-local & 132 & 168 \\
$\mathrm{RC}$ & Local & 117 & 183 \\
& Non-local & 112 & 188 \\
\hline
\end{tabular}

Table 1. Number of ta-ziji vs. ziji across conditions in Experiment 1. CP RC
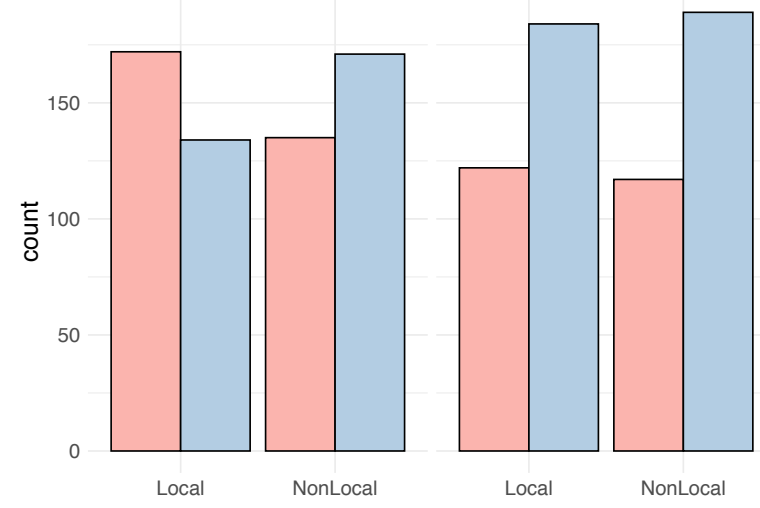

$\begin{array}{r}\text { Choice } \\ \square \text { Taziji } \\ \hline \square \text { Ziji }\end{array}$

Figure 2. Choice of ta-ziji vs. ziji across conditions in Experiment 1.

To assess these patterns statistically, we fit intercept-only mixed-effect logistic regression models with the proportion of $t a-z i j i$ choices as the dependent variable. Four models were fit, one for each of the four conditions. Statistical analyses were conducted in R (R Core Team 2018). A significant main effect of intercept would mean that the average proportion of $t a-z i j i$ is above $50 \%$ chance level. We reason that if the preference for $t a-z i j i$ is above chance, this would mean that $z i j i$ is significantly below chance (since the proportions of ziji and ta-ziji add up to $100 \%$ ) and thus participants preferred $t a-z i j i$ over $z i j i$; the opposite would be true if the proportion of $t a-$ $z i j i$ is below chance. Figure 3 displays the average proportion of $t a-z i j i$ across conditions with the same set of data used to plot Figure 2 . The $50 \%$ chance level is indicated by a blue line. 


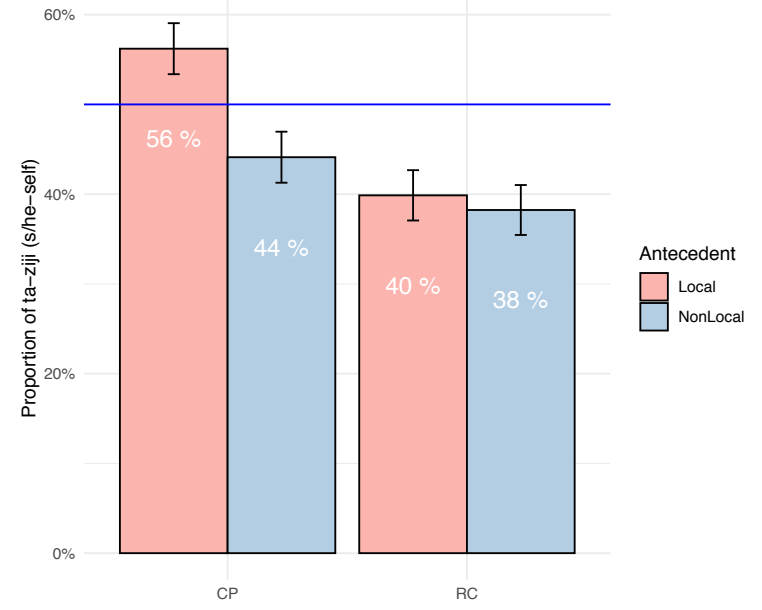

Figure 3. Proportions of ta-ziji across conditions in Experiment 1.

Statistical analyses show that in the $\mathrm{CP} /$ Local condition, the proportion of ta-ziji choices does not differ from chance, despite being numerically above $50 \%(\beta=0.29, \mathrm{SE}=0.18, \mathrm{t}=1.59, \mathrm{p}=$ 0.11). In the $\mathrm{CP} /$ Non-local condition, the proportion of ta-ziji choices also does not differ from chance $(\beta=-0.27, \mathrm{SE}=0.17, \mathrm{t}=-1.63, \mathrm{p}=0.10)$, despite being numerically below $50 \%$. In the two RC conditions, the proportion of ta-ziji choices is significantly below chance (RC/Local: $\beta=$ $-0.51, \mathrm{SE}=0.19, \mathrm{t}=-2.57, \mathrm{p}=0.01$; RC/Non-local: $\beta=-0.68, \mathrm{SE}=0.26, \mathrm{t}=-2.67, \mathrm{p}<0.01)$.

3.4. DISCUSSION. In Experiment 1, we asked Chinese natives to choose ziji or ta-ziji in genitive forms in non-strictly local CP structures and strictly local RC structures. Overall, the results of Experiment 1 show that native speakers' choice patterns vary depending on the structure type and the compatible antecedent position. In the CP structures, their judgment patterns hint at an asymmetric pattern such that they show a numerical preference for ta-ziji in local contexts and ziji in non-local contexts. This is in line with a view that $t a-z i j i$ is more constrained by Condition A than $z i j i$, although the numeric trends failed to reach significance. Intriguingly, for RC structures, participants choose ziji more often than ta-ziji, regardless of antecedent position. One possibility for the divergent choice patterns in CP and RC structures may be due to the complexity of RCs which arguably require more cognitive resources. Participants might choose the more frequent ziji to alleviate the processing burden. We address this issue in Experiment 2.

4. Experiment 2. In this experiment, we minimally changed the stimuli from Experiment 1 to include a pre-RC classifier phrase for RC structures in $(5 c, d)$. Pre-RC classifiers have been shown in previous studies to signal an upcoming head noun (HN) and can reduce processing burden (e.g., $\mathrm{Wu}$ 2009, Wu et al. 2009, 2014). As explained previously, the classifier ming and jia are only compatible with a human and non-human NP, respectively. Thus, in an RC structure, by the time a reader reaches the classifier ming $_{\text {HUMAN }}$ or $j i a_{N O N-H U M A N}$, even before the onset of the HN, she will be able to predict the animacy property (human/non-human, animate/inanimate) of the HN (strictly local antecedent). The benefit is that when the $\mathrm{HN}$ is encountered, it is partially anticipated as its animacy property has already been revealed by the classifier, thereby reducing the processing load for the reader.

4.1. PARTICIPANTS. Fifty-one participants self-identified as Mandarin native speakers (mean age $=22 ; \mathrm{SD}=2.80$ ) participated via Qualtrics. None had participated in Experiment 1. 
4.2. MATERIALS AND DESIGN. The materials are identical to Experiment 1 except that RC structures are preceded by demonstrative classifiers, shown in $(7 a, b)$ :

(7) a. RC structure/Local antecedent

Diantai biaoshi na-ming gongbu-le ___ caifang mudi de jizhe

Radio station say that-CL reveal-PERF interview purpose DE journalist yinqi-le dajia-de buman.

provoke-PERF everyone-GEN anger

'The radio station said that the journalist who revealed the purpose of voked everyone's anger.'

b. RC structure/Non-local antecedent

Jizhe biaoshi na-jia gongbu-le___ caifang mudi de diantai

journalist say that-CL reveal-PERF interview purpose DE radio station

yinqi-le dajia-de buman.

provoke-PERF everyone-GEN anger

'The journalist said that the radio station which revealed the purpose of interview provoked everyone's anger.'

4.3. RESULTS. Table 2 shows participants' choices of $t a-z j i$ and $z i j i$ across conditions (see Figure 4). In CP structures, $t a-z i j i$ was preferred over ziji in contexts with (non-strictly) local antecedents, while there was no clear preference in contexts with non-local antecedents. In RC structures, $t a-z i j i$ was again preferred in (strictly) local contexts, but ziji was preferred in non-local contexts.

\begin{tabular}{llll} 
Structure type & Antecedent position & ta-ziji & ziji \\
\hline $\mathrm{CP}$ & Local & 220 & 92 \\
& Non-local & 154 & 158 \\
$\mathrm{RC}$ & Local & 178 & 134 \\
& Non-local & 137 & 175 \\
\hline
\end{tabular}

Table 2. Number of ta-ziji vs. ziji across conditions in Experiment 2.

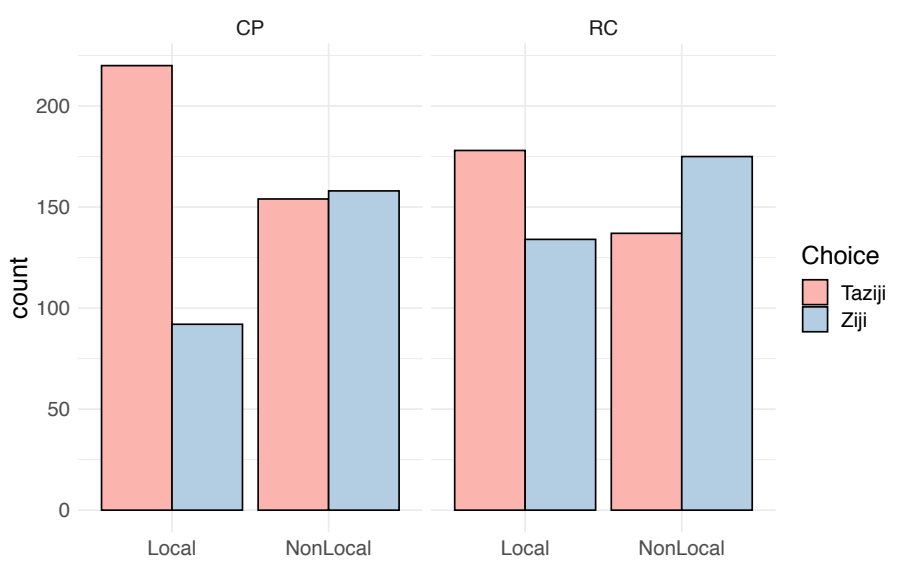

Figure 4. Choices of ta-ziji vs. ziji across conditions in Experiment 2.

To assess the (dis-)preferences for ta-ziji across conditions in Experiment 2, we similarly fit four mixed-effect intercept-only logistic models. As reasoned previously, if the proportion of $t a-$ $z i j i$ is above chance level, we take this as evidence that ta-ziji is preferred more than ziji; if the 
proportion of $t a-z i j i$ is below chance, we assume ziji is preferred. See Figure 5 for proportions of $t a-z i j i$ across conditions with the same data used for Figure 4.

Statistical analyses show that in the $\mathrm{CP} /$ Local structures, the proportion of ta-ziji choices is significantly above chance $(\beta=1.35, \mathrm{SE}=0.32, \mathrm{t}=4.26, \mathrm{p}<0.001)$. In the $\mathrm{CP} / \mathrm{Non}-$ local condition, the proportion of ta-ziji choices does not differ significantly from chance $(\beta=-0.03, \mathrm{SE}=$ $0.19, \mathrm{t}=-0.16, \mathrm{p}=0.88)$. In the $\mathrm{RC} /$ Local structures, the proportion of $t a-z i j i$ choices is marginally above chance $(\beta=0.34, \mathrm{SE}=0.18, \mathrm{t}=1.86, \mathrm{p}=0.06)$, but in the RC/Non-local structures, it is marginally below chance $(\beta=-0.28, \mathrm{SE}=0.17, \mathrm{t}=-1.69, \mathrm{p}=0.09)$.

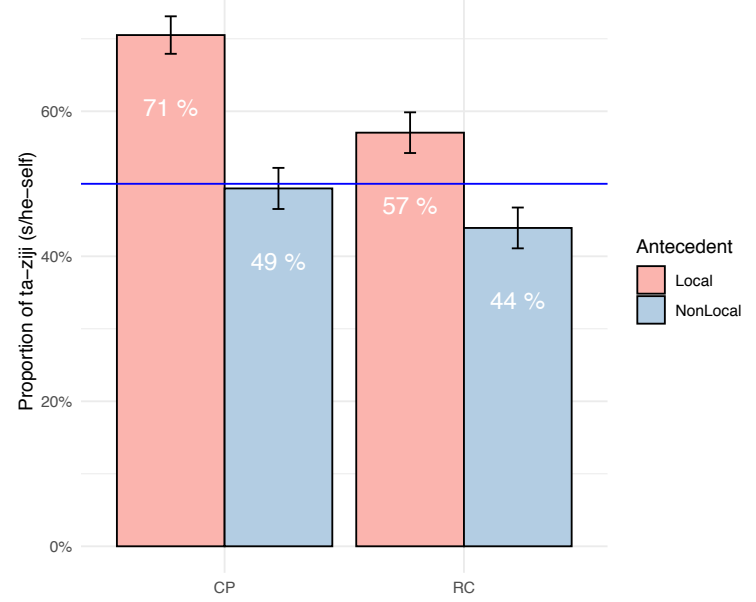

Figure 5. Proportions of ta-ziji across conditions in Experiment 2.

Interestingly, a visual comparison of Experiment 1 and 2 (Figure 3 and 5) seems to suggest that in Experiment 2, the proportions of ta-ziji across conditions increased overall. To verify this observation, we fit a mixed-effect logistic model with three factors, Structure type (coding: $\mathrm{CP}=$ $0.5, \mathrm{RC}=-0.5$ ), Antecedent position (coding: Local $=0.5$, Non-local $=-0.5$ ), and Experiment (coding: Exp. $1=0.5$, Exp. $2=-0.5)$. Statistical analysis revealed a main effect of Experiment $(\beta=$ $-0.53, \mathrm{SE}=0.19, \mathrm{t}=-2.65, \mathrm{p}<0.01$ ), suggesting that the average proportion of ta-ziji is lower in Experiment 1 than in Experiment 2. Notably, there is an interaction of Experiment and Antecedent position $(\beta=-0.51, \mathrm{SE}=0.18, \mathrm{t}=-2.87, \mathrm{p}<0.005)$, suggesting the increase of ta-ziji in local contexts compared to non-local contexts in Experiment 1 was boosted in Experiment 2.

4.4. DiSCUSSION. The results from Experiment 2 show that the stronger bias for ta-ziji in strictly local contexts is consistent across CP and RC structures. Although the bias for ziji in non-local contexts is only statistically meaningful in RC structures, the results are more in line with the view that $t a-z i j i$ is more local in nature and do not support an alternative view that $z i j i$ is more constrained by Condition A than ta-ziji.

In addition, we observed that the preference for ta-ziji increased overall in Experiment 2. As these two experiments only differ in the presence/absence of classifiers in RC structures, we link these divergent choice patterns to the increased presence of classifiers in Experiment 2. Specifically, we speculate that more frequent encounters of classifiers make local antecedents more salient because a classifier cue flags and reinforces the presence of the local antecedent. For example, the presence of a pre-RC classifier makes the processing of RC structures easier due to the predictability of the local HN (e.g., Wu 2009; Wu et al. 2014). The increased exposure to classifiers likely prompted participants to choose a reflexive that is more compatible with a local context. The fact that ta-ziji was selected more frequently as a result lends support to the view 
that $t a-z i j i$ is preferred in local contexts than ziji (if native speakers are not cognitively burdened as when pre-RC classifier cues are available to reduce the processing load).

5. General discussion. This study aims to examine which reflexive, ziji or ta-ziji, is more constrained by Condition A, motivated by the previous findings that both reflexives tend to be interpreted as syntactic reflexives whenever possible. We approach this question by using a forced choice task where native speakers were required to choose either ziji or ta-ziji in their genitive forms, in contexts with local or non-local antecedents. In addition to the regular locality design where the local antecedent linearly precedes the reflexive in a CP structure, we included conditions where the local antecedent can follow the reflexive as in a RC structure, thus disentangling syntactic locality from linear precedence.

In Experiment 1, we observed an asymmetric choice pattern in CP structures as ta-ziji was preferred in local contexts and ziji was preferred in non-local contexts, although statistics did not reach significance. Therefore, we only have weak evidence that ta-ziji is more local than ziji based on observation of numeric trends. However, it should be emphasized that the opposite pattern was not observed at all, that is, we see no evidence that ziji is preferred in local contexts and $t a-z i j i$ in non-local contexts. Therefore, while there is no strong evidence supporting ta-ziji as more local, there is no evidence for the opposite view either. Interestingly, in RC structures, the asymmetric pattern was not observed, which we speculated could be due to the complexity of $\mathrm{RCs}$, which prompted natives to favor ziji which is easier to retrieve from the mental lexicon.

In Experiment 2, we tried to alleviate the potential working memory burden associated with the processing of RCs by providing participants with a pre-RC classifier cue, as prior studies showed that pre-RC classifiers can reduce processing burden (e.g., Wu 2009, Wu et al. 2014). With this manipulation, an asymmetric choice pattern emerged in RC structures. Intriguingly, higher proportions of $t a-z i j i$ were found in Experiment 2 overall compared to Experiment 1 . We speculate that as classifiers pre-activate the local antecedent, more frequent encounters of classifiers in Experiment 2 made participants pay more attention to local antecedents. Consequently, participants considered more frequently the reflexive that fits better with a local environment. Since ta-ziji was considered more often as a result, we have additional evidence that ta-ziji is more local than ziji.

The above results do not support the hypothetical view inspired by previous findings that ziji shows stronger sensitivity to Condition A than ta-ziji. Recall that Dillon et al. (2016) found that in sentences where the reflexive is in a direct object position, ziji shows stronger locality bias than ta-ziji (also see Wang (2017a, b) for similar findings with ziji and ta-ziji in subject position). If the locality bias difference between $z i j i$ and $t a-z i j i$ stems from their different sensitivities to Condition A, we should observe ziji to be preferred in local contexts in this study, contrary to our findings. The differences between this study and prior ones ${ }^{2}$ may be related to the tasks that were used in the various experiments (e.g., acceptability judgments vs. selecting one form over the other). The differences may also have to do with how easily ta-ziji can be interpreted as being emphatic (cf. he himself in English). As mentioned above, emphatic ta-ziji is exempt from Condition A (Pan 1998). A higher proportion of emphatic readings of ta-ziji could account for the weaker locality bias of ta-ziji observed in prior work relative to our results. One way to examine this possibility would be to use spoken stimuli instead of written stimuli, so that the emphatic

\footnotetext{
${ }^{2}$ We acknowledge that individual variation might also account for the difference. Participants speaking different Chinese dialects could have different biases (see e.g., difference between Mandarin and Teochew (Cole et al. 2001)). As we have not looked into the influence of dialects in this work, we will not speculate any further.
} 
reading of ta-ziji can be ruled out by the non-emphatic intonation. More work is needed to clarify the locality bias question.

Finally, we briefly comment on the theoretical contributions of the present work. Firstly, if we follow prior experimental work by assuming that ziji is by default a syntactic reflexive (but see our note above regarding Charnavel 2020), then the present work suggests that Condition A has different weights on the interpretation of ziji and ta-ziji, as the morphologically complex $t a-$ $z i j i$ seems to be more constrained by Condition A. In other words, the influence of Condition A on anaphora resolution is form-specific. Therefore, even if one holds the view that both ziji and ta-ziji are syntactic reflexives by default, our results suggest that this does not mean that they are equally constrained by Condition A. However, it is important to acknowledge that if we view ziji as ambiguous between a syntactic reflexive and an exempt reflexive sensitive to perspective-taking, we also expect it to show lower sensitivity to Condition A since it would not be governed by Condition A when exempt. We leave these issues for future work.

Secondly, we see no evidence that ziji is replacing $t a-z i j i$ as a syntactic reflexive, as suggested earlier. Native speakers still prefer to associate ta-ziji with local contexts. This indicates that the two reflexives still overlap in their functions at the current stage of lexical evolution in Mandarin Chinese. However, according to the mutual competitive exclusion account (Lindsay \& Aronoff 2013; Aronoff 2019), ultimately, each reflexive will have its own functional niche. The fact that $z i j i$ and $t a-z i j i$ do not have their own division of labor yet does not mean that they will not do so in the future. In fact, one way to verify the competitive mutual exclusion account in the context of bare and compound reflexives might be to look at the equivalents of ziji and ta-ziji in other Chinese dialects. We plan to look into this in our future work.

6. Conclusion. In two forced choice judgment experiments, we investigated Chinese native speakers' preference for ziji and ta-ziji in sentences with local and non-local antecedents. Combined experimental results from these two experiments fit better with claims that in local contexts, $t a-z i j i$ is preferred over $z i j i$. We find no overarching evidence of ziji being preferred over ta-ziji in local contexts. As a whole, these results do not support a hypothetical view that ziji is more constrained by Condition A than ta-ziji. In fact, the results are more in line with the opposite view. Although not consistent with the results from some prior studies, the results from this study, together with results from earlier ones, support a form-specific view of anaphora resolution.

\section{References}

Aronoff, Mark. 2019. Competitors and alternants in linguistic morphology. In Franz Rainer, Francisco Gardani, Wolfgang U. Dressler \& Hans C. Luschützky (eds.), Competition in inflection and word-formation. Studies in Morphology 5. Cham: Springer.

Charnavel, Isabelle, Peter Cole, Gabriella Hermon \& C.-T. James Huang. 2017. Long-distance anaphora: Syntax and discourse. In Martin Everaert \& Henk C. van Riemsdijk (eds.), The Wiley Blackwell Companion to Syntax, 2321-2402. Hoboken, NJ: John Wiley \& Sons, Inc. https://doi.org/10.1002/9781118358733.wbsyncom074.

Charnavel, Isabelle. 2020. Logophoricity and locality: A view from French anaphors. Linguistic Inquiry, 51(4). 671-723. https://doi.org/10.1162/ling_a_00349.Chomsky,

Noam. 1981. Lectures on government and binding. Dordrecht: Foris.

Chou, Xiaoli. 1992. An alternative to Chinese reflexives. Austin, TX: University of Texas M.A. thesis. 
Cole, Peter, Gabriella Hermon \& C. Leng Lee. 2001. Grammatical and discourse conditions on long distance reflexives in two Chinese dialects. In Peter Cole, Gabriella Hermon \& C.-T. James Huang (eds.), Long distance reflexives, 141-195. New York: Academic Press. https://doi.org/10.1163/9781849508742_002.

Dillon, Brian, Wing-Yee Chow \& Ming Xiang. 2016. The relationship between anaphor features and antecedent retrieval: Comparing Mandarin ziji and ta-ziji. Frontiers in Psychology 6. 1966. https://doi.org/10.3389/fpsyg.2015.01966.

Dillon, Brian, Wing-Yee Chow, Matthew Wagers, Taomei Guo, Fengqin Liu \& Colin Phillips. 2014. The structure-sensitivity of memory access: Evidence from Mandarin Chinese.

Frontiers in Psychology, 5. 1025. https://doi.org/10.3389/fpsyg.2014.01025.

Gao, Liqun, Zhaojing Liu \& Yueyuan Huang. 2005. Who is ziji? An experimental study about the binding principle. Linguistic Sciences 4. 39-50.

Huang, C.-T. James, Y.-H. Aaudrey Li \& Yafei Li. 2009. The syntax of Chinese. Cambridge: Cambridge University Press.

Huang, C.-T. James, \& C.-S. Luther Liu. 2001. Logophoricity, attitudes, and ziji at the interface. In Peter Cole, Gabriella Hermon \& C.-T. James Huang, (eds.), Long-distance reflexives, 141-195. New York: Academic Press.

Jäger, Lena A., Felix Engelmann \& Shravan Vasishth. 2015. Retrieval interference in reflexive processing: Experimental evidence from Mandarin, and computational modeling. Frontiers in Psychology, 6. 617. https://doi.org/10.3389/fpsyg.2015.00617.

Kaiser, Elsi \& John C. Trueswell. 2008. Interpreting pronouns and demonstratives in Finnish: Evidence for a form-specific approach to reference resolution. Language and Cognitive Processes 23(5). 709-748. https://doi.org/10.1080/01690960701771220.

Kuno, Susumu. 1972. Pronominalization, reflexivization and direct discourse. Linguistic inquiry, 3(2). 161-195.

Kuno, Susumu. 1987. Functional syntax: Anaphora, discourse, and empathy. Chicago: The University of Chicago Press.

Li, Xiaoqian \& Xiaolin Zhou. 2010. Who is ziji? ERP responses to the Chinese reflexive pronoun during sentence comprehension. Brain \& Research, 1331. 96-104. https://doi.org/10.1016/j.brainres.2010.03.050.

Lindsay, Mark \& Mark Aronoff. 2013. Natural selection in self-organizing morphological systems. Morphology in Toulouse: Selected Proceedings of Décembrettes 7. 133-153.

Lu, Hsin-Yi. 2011. The effects of verb bias, context, and tasks on Mandarin Chinese Reflexives. Champaign, IL: University of Illinois dissertation.

Pan, Haihua. 1995. Locality, self-ascription, discourse prominence, and Mandarin reflexives. Austin, TX: University of Texas dissertation.

Pan, Haihua. 1997. Constraints on reflexivization in Mandarin Chinese. New York: Garland.

Pan, Haihua. 1998. Closeness, prominence, and binding theory. Natural Language \& Linguistic Theory 16(4). 771-815. https://doi.org/10.1023/A:1006056414208 .

Pollard, Carl \& Ivan A. Sag. 1992. Anaphors in English and the scope of Binding Theory. Linguistic Inquiry 23(2). 261-303. https://www.jstor.org/stable/4178768.

Postal, Paul. 1971. Crossover phenomena. New York: Holt, Reinhart and Winston.

R Core Team. 2018. R: A language and environment for statistical computing. https://www.R-project.org/.

Reinhart, Tanya \& Eric J. Reuland. 1993. Reflexivity. Linguistic Inquiry 24(4). 657-720. https://www.jstor.org/stable/4178836. 
Sells, Peter. 1987. Aspects of logophoricity. Linguistic Inquiry 18(3). 445-479. https://www.jstor.org/stable/4178550.

Wang, Y. \& Haihua Pan. 2015a. Empathy and Chinese long-distance reflexive ziji - Remarks on Giorgi (2006, 2007). Natural Language \& Linguistic Theory 33. 307-322. https://doi.org/10.1007/s11049-014-9257-5.

Wang, Yuxia. 2017a. Semantic-constrained or syntactic-constrained? - Processing Chinese bare and compound reflexives. Journal of Psychological Science 40. 527-533.

Wang, Yuxia. 2017b. An eye-movement study on the online processing of Chinese bare and compound reflexives. Journal of SJTU (Philosophy and Social Sciences) 25(4). 97-106.

Wu, Fuyun, Elsi Kaiser \& Elaine Anderson. 2009. The effects of classifiers in predicting Chinese relative clauses. In Michael Grosvald \& Dionne Soares (eds.), Proceedings of the Western Conference on Linguistics (WECOL), 318-329. Davis, CA: University of California.

Wu, Fuyun, Yingyi Luo \& Xiaolin Zhou. 2014. Building Chinese relative clause structures with lexical and syntactic cues: Evidence from visual world eye-tracking and reading times. Language, Cognition and Neuroscience, 29(10). 1205-1226. https://doi.org/10.1080/01690965.2013.841969.

Wu, Fuyun. 2009. Factors affecting relative clause processing in Mandarin. Los Angeles: University of Southern California dissertation.

$\mathrm{Xu}$, Liejiong. 1993. The long-distance binding of ziji. Journal of Chinese Linguistics 21. 123141.

Zribi-Hertz, Anne. 1989. Anaphor binding and narrative point of view: English reflexive pronouns in sentence and discourse. Language 65(4). 695-727. https://doi.org/10.2307/414931. 